\title{
Associations between cardiovascular risk factors and psoriasis in Iran
}

This article was published in the following Dove Press journal:

Clinical, Cosmetic and Investigational Dermatology

10 August 2015

Number of times this article has been viewed

\author{
Mahmoud Farshchian \\ Akram Ansar \\ Mohammadreza Sobhan \\ Psoriasis Research Center, \\ Department of Dermatology, \\ Farshchian Hospital, Hamadan \\ University of Medical Sciences, \\ Hamadan, Iran
}

Correspondence: Mohammadreza Sobhan Psoriasis Research Center, Department of Dermatology, Farshchian Hospital, Hamadan University of Medical Sciences, Mirzade Eshghy Street, Hamadan 65I6840874I, Iran

Tel +989121089455

Fax +988138269808

Email mreza_sobhan@yahoo.com
Background: Psoriasis is a common chronic inflammatory skin disease. There is overwhelming evidence on the higher risk of cardiovascular diseases in patients with psoriasis as a result of hyperlipidemia, which is more common in these patients.

Objectives: The aim of this study was to elucidate the association between the cardiovascular risk factors and psoriasis.

Methods: In a cross-sectional study, 55 patients with psoriasis and 55 matched (sex and age) controls were entered the study at the Department of Dermatology between March 2011 and March 2013. Blood samples were obtained following 14 hours fasting status and serum levels of triglyceride, cholesterol, high-density lipoprotein, and low-density lipoprotein were determined using standard laboratory methods, and other variables such as sex, age, smoking, alcohol consumption, and the type of disease were recorded.

Results: Our findings showed that levels of triglyceride, low-density lipoprotein, and smoking were significantly higher in psoriatic patients when compared with controls, whereas the level of high-density lipoprotein and cholesterol was not significantly different between two groups. Body mass index of psoriatic patients was not significantly higher than controls. Patients with psoriasis also had an increased prevalence of hypertension.

Conclusion: Our findings further verify lipid abnormalities in psoriatic patients. Psoriasis is associated with higher rate of hypertension, which may be resulted in increasing the risk of cardiovascular diseases in these patients. Thus, serum lipid profile and blood pressure in all patients with psoriasis, regardless of disease severity, deserve consideration to be checked.

Keywords: cardiovascular disease, risk factors, psoriasis, lipid profile

\section{Introduction}

Psoriasis is a common, chronic, T-lymphocyte-mediated inflammatory skin condition characterized by scaly erythematosus plaques on body surfaces, which affects nearly $2 \%-3 \%$ of the world's population in all geographic regions. ${ }^{1}$ The prevalence rates reported from Africa and Asia varied from no cases detected to estimates below $0.5 \%{ }^{2}$ Psoriasis lesions have a predilection for nails, scalp, genitalia, extensor surfaces, and the lumbosacral region. ${ }^{1}$ To date, extradermatic manifestations of psoriasis have been well characterized. Also emerging associations between psoriasis and other systemic diseases such as obesity, diabetes mellitus, depression or addiction, cancer, chronic inflammatory bowel disease, arthritis, and cardiovascular diseases (CVD) have been reported. ${ }^{3-6}$ Some authors pointed out that psoriasis patients require education about their increased CVD risk factors. There is a need to train dermatologists about the risk factors of systemic diseases, which should be examined regularly in diseased patients. ${ }^{7}$ 
CVD risk has been shown to be higher in younger patients and those with more severe psoriasis. ${ }^{4,5}$ Since psoriasis is associated with smoking and alcohol intake, the increase in CVD and mortality among psoriatic patients is thought to be compounded by the cumulative effect of traditional risk factors for CVD. ${ }^{1}$ However, a cohort study showed that severe psoriasis is found to be a significant independent risk factor for cardiovascular mortality even after adjusting for traditional CVD risk factors. ${ }^{8}$ Although the increased prevalence of myocardial infarction varies with age and disease severity, it remains significantly elevated after controlling for traditional cardiovascular risk factors. ${ }^{5}$ Association of CVD and psoriasis is controversial. In some studies, the risk of CVD has been reported to be higher in patients with psoriasis. ${ }^{1,5,9-17}$ However, in some other reports in overall examination and considering the whole psoriatic patients the risk of CVD, transient ischemic attacks, or cerebrovascular accidents remained unchanged between psoriatic patients and controls. ${ }^{18}$ Furthermore, no association between psoriasis and metabolic syndrome has been observed in Korean psoriatic patients. ${ }^{19}$ Another considerable finding is the lack of significant difference of the ischemic heart disease risk between psoriatic patients and controls in a large populationbased Dutch cohort. ${ }^{20}$

On the basis of a retrospective and cross-sectional study, the prevalence of papulosquamous diseases such as psoriasis and lichen planus was reported 15 per 10,000 population in rural areas of Hamadan, Iran. ${ }^{21}$ One major question to be considered is whether Iranian patients of psoriasis are susceptible to metabolic syndrome risk factors and CVD. Here, we have studied the associations of CVD and metabolic syndrome risk factors with psoriasis in a population of Iranian psoriatic patients.

\section{Methods}

\section{Study population}

A case-control study of age- and sex-matched individuals with 55 psoriatic patients and 55 controls was performed. Histopathologically confirmed psoriatic patients with 20 years of age or older visited the Department of Dermatology, including both in- and out-patients, Farshchian Hospital, Hamadan, Iran; between March 2011 and March 2013 were entered the study. Control group consisted of the healthy individuals accompanying the patients at the Department of Dermatology between March 2011 and March 2013, with no history of immunodeficiency, immunosuppressive medications, and dermatologic diseases. A written informed consent was signed by every patient following explanation of the nature of the study. All individuals were interviewed by a dermatologist and underwent physical examinations. Information regarding the smoking status and alcohol consumption, and medical problems including CVD, hypertension, diabetes, and hyperlipidemia, were recorded. In addition, family history of CVD; and duration, extent, and severity of psoriasis, height, weight, and blood pressure (BP) were measured for all subjects.

\section{Measurements}

A fasting blood sample (3 cc) was obtained from each participant and underwent centrifugation at 3,000 rpm for 10 minutes to separate serum. The serum was then analyzed by chemical assay kits (Pars Azmoon, Tehran, Iran) with Hitachi 902 auto analyzer to measure triglyceride (TG), high-density lipoprotein (HDL), and low-density lipoprotein (LDL). The following values were considered normal: $\mathrm{TG}<200 \mathrm{mg} / \mathrm{dL}$; cholesterol total $<200 \mathrm{mg} / \mathrm{dL} ; \mathrm{LDL}<160 \mathrm{mg} / \mathrm{dL}$; HDL $>35$ $\mathrm{mg} / \mathrm{dL}$ in men; and HDL $>45 \mathrm{mg} / \mathrm{dL}$ in women; fasting blood glucose $(\mathrm{FBS})<126 \mathrm{mg} / \mathrm{dL}$.

\section{Ethical approval}

This study was approved by the National Research Council of the Islamic Republic of Iran and was performed with the approval of the ethics human research review committee of the Hamadan University of Medical Sciences. The study was performed according to the Declaration of Helsinki.

\section{Statistical analysis}

The statistical analysis was performed in two steps. First, we used chi-square test and Student's $t$-test to determine the statistical significance of the differences of age, sex, smoking, obesity, CVD, hypertension, diabetes, hyperlipidemia, and metabolic syndrome between the patients with psoriasis and the controls. The mean and standard deviation were used to report the data. In the second step, chi-square tests and logistic regression analysis were used to determine the risk factors for those significant differences of first step. Odds ratios (OR) and the level of significance ( $P$-values) are reported. All these statistical investigations were performed by SPSS (Windows version 16.00; SPSS Inc., Chicago, IL, USA). A $P$-value of $<0.05$ was considered as statistically significant.

\section{Results}

The mean age of individuals in psoriatic patients was 47.3 $( \pm 18.4)$ and in control group was $45.16( \pm 15.9)$. In patients group, $65.5 \%$ of cases $(n=36)$ and in control group $64 \%$ $(n=35)$ were male. No significant difference was observed 
for age and sex between groups. In psoriatic patients, $85 \%$ $(n=47)$ had plaque type, $2 \%(n=1)$ had guttate type, $9 \%(n=5)$ had plaque and guttate type, and $4 \%(n=2)$ had pustules and guttate type. Smoking status was significantly $(P=0.03)$ different between cases $(n=32 ; 58.2 \%)$ and controls $(n=9$; $16.4 \%$ ). Alcohol consumption was observed in nine psoriatic patients while no controls were alcohol users. Differences between cases and controls regarding aforesaid measured variables are displayed in Table 1. Body mass index (BMI) of cases $(26.36 \pm 4.71)$ was significantly $(P=0.02)$ higher than patients $(24.6 \pm 3)$. Interestingly, LDL and TG of psoriatic patients were significantly higher $(\mathrm{LDL}, P=0.01$; TG, $P<0.001)$ compared with controls. In addition, we observed that systolic and diastolic BPs of cases were significantly higher (systolic BP, $P<0.001$; diastolic $\mathrm{BP}, P=0.003$ ) compared with controls. Although the mean of FBS and HDL levels was higher in cases as compared with controls, these differences were not statistically significant.

Multivariate logistic regression analysis was performed to analyze the association between psoriasis and risk factors of CVD and metabolic syndrome (Table 2). In final logistic regression model, systolic BP, LDL, TG, and smoking were statistically significant. The results of the analysis revealed that psoriasis was associated with systolic hypertension and elevated LDL and TG. Patients with psoriasis had an increased prevalence of systolic hypertension (OR 1.07, 95\% confidence interval [CI] 1.02-1.33). A significant relationship ( $P=0.02$ ) was found between smoking status and psoriasis $(\mathrm{OR}=2.2 ; \mathrm{CI} \% 95=1.1-3.2)$. Also TG and psoriasis $(\mathrm{OR}=1.02 ; \mathrm{CI} \%$ 95=1-1.34) showed a significant association $(P=0.005)$. The other considerable finding was the significant relationship $(P=0.03)$ between LDL and psoriasis $(\mathrm{OR}=1.04 ; \mathrm{CI} \% 95=1-1.09)$ and in psoriasis the level of LDL was fourfolds higher. No significant association was observed between BMI and disease.

\section{Discussion}

This study was performed in a population of Iranian psoriatic patients to find out the association between CVD risk factors and psoriasis. We observed that serum TG and LDL are significantly higher in psoriatic patients, which augmented the risk of CVD independent of disease severity. In addition, we found that systolic BPs in patients were significantly higher than controls. These findings, in addition to increased smoking, describe a considerable alarm for cardiovascular events in Iranian psoriatic patients.

The comparisons between cases and controls revealed no significant difference between BMI of cases and controls. In accordance with our findings, Herron et al found no significant difference between BMI of 500 female psoriatic patients and controls. ${ }^{22}$ Conversely, Madanagobalane and Anandan found a significant difference in BMI between 118 South Indian psoriasis vulgaris patients and 120 controls. ${ }^{23}$

Total cholesterol of psoriatic patients was not significantly higher compared with controls in our study. This finding was not in agreement with the previous study performed by Pietrzak et al in which there was a significant difference between total cholesterol of psoriatic patients and controls. ${ }^{24}$ In addition, Yaghmaei and Fekri found a significant difference between total cholesterol of psoriatic patients $(177.65 \mathrm{mg} / \mathrm{dL})$ and controls $(170.21 \mathrm{mg} / \mathrm{dL}) .{ }^{25}$ Dreiher et al conducted a study that included 10,669 psoriatic patients and 22,996 controls. It revealed that psoriasis was associated with TG and HDL but the association was not statistically significant for total cholesterol or LDL. ${ }^{26}$ Increasing evidence supports that psoriasis exfoliative lesions contain higher levels of cholesterol and lower free fatty acid concentrations compared with normal skin, accordingly in active phase of psoriasis in which shedding of skin (exfoliation) occurs, abundant cholesterol levels are lost that can increase the stimulation of the cholesterol synthesis. ${ }^{27}$ However, Farshchian et al in their study

Table I Univariate analysis for cardiovascular risk factors in psoriasis patients compared with the control group

\begin{tabular}{llll}
\hline Variable & Psoriasis patients (mean \pm SD) & Controls (mean \pm SD) & $P$-value \\
\hline Age & $47.30 \pm 18.40$ & $45.16 \pm 15.90$ & 0.5 \\
TG & $152.60 \pm 72.00$ & $107.05 \pm 35.00$ & $P<0.001$ \\
HDL & $66.00 \pm 10.40$ & $49.00 \pm 9.00$ & 0.1 \\
LDL & $106.00 \pm 36.00$ & $89.80 \pm 31.30$ & 0.01 \\
BMI & $26.36 \pm 4.71$ & $24.60 \pm 3.00$ & 0.02 \\
Diastolic BP & $81.11 \pm 11.30$ & $74.04 \pm 10.90$ & 0.003 \\
Systolic BP & $135.83 \pm 19.00$ & $118.10 \pm 16.00$ & $P<0.001$ \\
Cholesterol total & $184.00 \pm 47.10$ & $165.00 \pm 38.00$ & 0.02 \\
FBS & $101.00 \pm 25.70$ & $96.00 \pm 14.40$ & 0.1 \\
\hline
\end{tabular}

Abbreviations: TG, triglyceride; HDL, high-density lipoprotein cholesterol; LDL, low-density lipoprotein cholesterol; BMI, body mass index; BP, blood pressure; FBS, fasting blood glucose. 
Table 2 Final multivariable logistic regression model for assessment of determined variables

\begin{tabular}{|c|c|c|c|}
\hline Variable & Odds ratio & $P$-value & $\begin{array}{l}95 \% \text { confidence } \\
\text { interval }\end{array}$ \\
\hline Smoking & 2.20 & 0.02 & I. $1-3.2$ \\
\hline Triglyceride & 1.02 & 0.005 & $\mathrm{I}-\mathrm{I} .34$ \\
\hline Total cholesterol & 0.96 & 0.60 & $0.001-0.93$ \\
\hline LDL (mg/dL) & 1.04 & 0.03 & $1-1.09$ \\
\hline $\mathrm{BMI}\left(\mathrm{kg} / \mathrm{m}^{2}\right)$ & 1.10 & 0.07 & $0.33-2.1$ \\
\hline Systolic BP & 1.07 & 0.004 & $1.02-1.33$ \\
\hline Diastolic BP & 0.96 & 0.32 & $0.89-1.03$ \\
\hline
\end{tabular}

Abbreviations: LDL, low-density lipoprotein cholesterol; BMI, body mass index; $\mathrm{BP}$, blood pressure.

on psoriasis showed that lipid profile in Iranian patients with psoriasis did not differ compared with controls. ${ }^{28}$

TG levels of psoriatic patients in our study were significantly higher $(P<0.001)$ compared with controls, which is in line with the study of Seishima et $\mathrm{al}^{29}$ and Yaghmaei and Fekri. ${ }^{25}$ Furthermore, Azizzadeh et al have found a significant difference between TG of psoriatic patients $(152 \mathrm{mg} / \mathrm{dL})$ and controls $(114 \mathrm{mg} / \mathrm{dL})$ while they did not find a significant correlation between serum lipid profile and onset age of disease. ${ }^{30}$ Moreover, Madanagobalane and Anandan found a significant difference in TG levels between 118 South Indian psoriasis vulgaris patients and 120 controls, but did not find a significant difference between HDL or BP of patients and controls. ${ }^{23}$ In a study of 479 Korean psoriatic patients and controls performed by Kim et al between 1999 and 2009, a significant difference in TG was found; while other indexes of metabolic syndrome such as obesity, BP, FBS, and HDL were not significantly different between patients and controls. ${ }^{19}$

It is of particular importance to note that LDL of psoriatic patients was significantly higher $(P=0.01)$ than controls in our study, which is line with the findings of Azizzadeh et al. ${ }^{30}$ However, Yaghmaei and Fekri found no significant difference for LDL between cases and controls. ${ }^{25}$

We did not find significant difference between HDL of patients and controls, which agrees with the studies of Madanagobalane and Anandan ${ }^{23}$ and Kim et al. ${ }^{19}$ In addition, Mallbris et al found no significant difference between HDL of cases and controls. ${ }^{31}$ However, Shahidi et al found significantly lower levels of HDL in psoriatic patients than controls..$^{32} \mathrm{~A}$ survey showed that decreased HDL was the main biological abnormality in Tunisian psoriatic patients. ${ }^{33}$

Our study investigated the risk of psoriasis and smoking, showing a significant relationship so that smoking increased the psoriasis risk nearly twofolds. More studies are needed to confirm this association as a causal relationship; meanwhile, it has been surveyed in some studies with probable confirmed associations. To provide an example, Herron et al showed that smoking intensity influences the risk of psoriasis more than smoking duration. They reported that smoking 20 cigarettes daily increases the risk of psoriasis threefolds, but such an effect has not been observed for obesity and BMI. ${ }^{22}$ Naldi et al showed that smoking was strongly associated with pustular psoriatic lesions. ${ }^{34}$ Ashkevari et al found a significant association between smoking and psoriasis, and smoking was noted to increase the psoriasis risk nearly twofolds. ${ }^{35}$ In addition, Gelfand et al observed a significant association between smoking and psoriasis. ${ }^{36}$ From a pathophysiological point of view, epidermal cell proliferation, differentiation, angiogenesis, and cellular immune system are seemed to be involved. Moreover, cholinergic receptors of cigarette nicotine on keratinocytes stimulate inflow of calcium and cell differentiation. Furthermore, smoking and cigarette nicotine may influence immune process in skin of psoriatic patients. ${ }^{37}$ On the basis of the findings of the present study, smokers with psoriasis should be encouraged to quit smoking. Our study showed a significant difference between alcohol consumption of psoriatic patients and controls in univariate analysis, while it was not confirmed in final model of logistic regression. Despite controversial findings regarding alcohol consumption and psoriasis risk, a recent meta-analysis showed that there is a significant association between psoriasis and alcohol consumption..$^{38}$ Altogether, the biologic role of alcohol in psoriasis requires further studies.

We found that systolic BP of cases was significantly higher in comparison with controls. Meta-analysis of Armstrong et al on observational studies elucidated that the risk of systolic hypertension is two times higher in psoriatic patients as compared with normal population, and also the more severe psoriasis develops, the more hypertension risk increases. ${ }^{39}$ In addition, Cohen et al observed an association between psoriasis and hypertension and suggested routine screening for hypertension in psoriasis patients. ${ }^{40}$ While Madanagobalane and Anandan reported no differences in BP of psoriatic patients and controls. ${ }^{23}$

FBS levels of psoriatic patients and controls were not significantly different in the present study, which is in accordance with the study of Kim et al. ${ }^{19}$ However in some studies, such as Ahlehoff et $\mathrm{al}^{41}$ and Pereira et al, ${ }^{42}$ a significantly higher FBS was observed in psoriatic patients as compared with controls. In a German cross-sectional study, psoriasis was significantly associated with type 2 diabetes and myocardial infarction. ${ }^{43}$

The small sample size could be considered as on of the limitations of the present study. We limited the inclusion 
criteria to individuals with 20 years of age or older. Thereby, the results cannot be generalized to psoriasis with an earlier onset. In addition, the data of smoking and alcohol consumption habit might be underreported due to the cultural issues.

\section{Conclusion}

In summary, the serum TG and LDL are significantly higher in cases resulted in increased risk of CVD independent of disease severity. Thus, the screening of hyperlipidemia in psoriatic patients would be recommended. In addition, we found the elevated BP of psoriatic patients compared with controls, which intrigues cardiologists and internists regarding screening and education of these patients for self-assessment of BP. The significant difference between smoking of cases and controls indicates that smoking might be an independent risk factor for psoriasis. Patients should be encouraged to quit smoking for a better prognosis of the disease. However, more comprehensive studies with larger sample sizes are required. Generally, the data in the present study are sufficient only for the suggestions, because recommendation of these screenings requires data from longitudinal prospective study.

\section{Disclosure}

The authors report no conflicts of interest in this work.

\section{References}

1. Lee $\mathrm{S}, \mathrm{Wu}$ J. A review of the epidemiology of cardiovascular comorbidities in psoriasis. Curr Dermatol Rep. 2012;1(1):14-22.

2. Parisi R, Symmons D, Griffiths C, Ashcroft D. Global epidemiology of psoriasis: a systemic review of incidence and prevalence. $J$ Invest Dermatol. 2013;133:377-385.

3. Kimball AB, Gladman D, Gelfand JM, et al. National Psoriasis Foundation clinical consensus on psoriasis comorbidities and recommendations for screening. J Am Acad Dermatol. 2008;58(6):1031-1042.

4. Prodanovich S, Kirsner RS, Kravetz JD, Ma F, Martinez L, Federman DG. Association of psoriasis with coronary artery, cerebrovascular, and peripheral vascular diseases and mortality. Arch Dermatol. 2009; 145(6):700-703.

5. Richard MA, Barnetche T, Horreau C, et al. Psoriasis, cardiovascular events, cancer risk and alcohol use: evidence-based recommendations based on systematic review and expert opinion. $J$ Eur Acad Dermatol Venereol. 2013;27(Suppl 3):2-11.

6. Vena GA, Vestita M, Cassano N. Psoriasis and cardiovascular disease. Dermatol Ther. 2010;23(2):144-151.

7. Lee MK, Kim HS, Cho EB, Park EJ, Kim KH, Kim KJ. A study of awareness and screening behavior of cardiovascular risk factors in patients with psoriasis and dermatologists. Ann Dermatol. 2015;27(1):59-65.

8. Mehta NN, Azfar RS, Shin DB, Neimann AL, Troxel AB, Gelfand JM. Patients with severe psoriasis are at increased risk of cardiovascular mortality: cohort study using the General Practice Research Database. Eur Heart J. 2010;31(8):1000-1006.

9. Mehta NN, Yu Y, Pinnelas R, et al. Attributable risk estimate of severe psoriasis on major cardiovascular events. Am J Med. 2011;124(8):775. e1-e6.
10. Ryan C, Menter A. Psoriasis and cardiovascular disorders. G Ital Dermatol Venereol. 2012;147(2):179-187.

11. Sommer DM, Jenisch S, Suchan M, Christophers E, Weichenthal M. Increased prevalence of the metabolic syndrome in patients with moderate to severe psoriasis. Arch Dermatol Res. 2006;298(7):321-328.

12. Tablazon IL, Al-Dabagh A, Davis SA, Feldman SR. Risk of cardiovascular disorders in psoriasis patients: current and future. Am J Clin Dermatol. 2013;14(1):1-7.

13. Ma L, Li M, Wang H, Li Y, Bai B. High prevalence of cardiovascular risk factors in patients with moderate or severe psoriasis in northern China. Arch Dermatol Res. 2014;306(3):247-251.

14. Parodi A, Aste N, Calvieri C, et al. Metabolic syndrome prevalence in psoriasis: a cross-sectional study in the Italian population. Am J Clin Dermatol. 2014;15(4):371-377.

15. Karoli R, Fatima J, Shukla V, et al. A study of cardio-metabolic risk profile in patients with psoriasis. J Assoc Physicians India. 2013; 61(11):798-803.

16. Edson-Heredia E, Zhu B, Lefevre C, et al. Prevalence and incidence rates of cardiovascular, autoimmune, and other diseases in patients with psoriatic or psoriatic arthritis: a retrospective study using Clinical Practice Research Datalink. J Eur Acad Dermatol Venereol. 2015;29(5):955-963.

17. Baeta I, Gontijo B, Bittencourt F, Goulart E. Comorbidities and cardiovascular risk factors in patients with psoriasis. An Bras Dermatol. 2014;89(5):735-744.

18. Brauchli YB, Jick SS, Miret M, Meier CR. Psoriasis and risk of incident myocardial infarction, stroke or transient ischaemic attack: an inception cohort study with a nested case-control analysis. Br J Dermatol. 2009;160(5):1048-1056.

19. Kim GW, Park HJ, Kim HS, et al. Analysis of cardiovascular risk factors and metabolic syndrome in Korean patients with psoriasis. Ann Dermatol. 2012;24(1):11-15.

20. Wakkee M, Herings RM, Nijsten T. Psoriasis may not be an independent risk factor for acute ischemic heart disease hospitalizations: results of a large population-based Dutch cohort. J Invest Dermatol. 2009; 130(4):962-967.

21. Zamanian A, MahjubH. Prevalence of skin diseases in Hamedan, Iran in 2002. Indian J Dermatol. 2005;50:208-211.

22. Herron MD, Hinckley M, Hoffman MS, et al. Impact of obesity and smoking on psoriasis presentation and management. Arch Dermatol. 2005;141(12):1527-1534.

23. Madanagobalane S, Anandan S. Prevalence of metabolic syndrome in South Indian patients with psoriasis vulgaris and the relation between disease severity and metabolic syndrome: a hospital-based case-control study. Indian J Dermatol. 2012;57(5):353-357.

24. Pietrzak AT, Zalewska A, Chodorowska G, et al. Cytokines and anticytokines in psoriasis. Clin Chim Acta. 2008;394(1):7-21.

25. Yaghmaei B, Fekri A. Lipid profile change in psoriatic patients. Kerman Med Sci J. 1994;1:17-22.

26. Dreiher J, Weitzman D, Davidovici B, et al. Psoriasis and dyslipidaemia: a population-based study. Acta Derm Venereol. 2008;88:561-565.

27. Lea WAJ, Cornish HH, Block WD. Studies on serum lipids, proteins, and lipoproteins in psoriasis. J Invest Dermatol. 1958;30(4):181-185.

28. Farshchian M, Zamanian A, Farshchian M, Monsef AR, Mahjub H. Serum lipid level in Iranian patients with psoriasis. J Eur Acad Dermatol Venerol. 2007;21(6):802-805.

29. Seishima M, Seishima M, Mori S, Noma A. Serum lipid and apolipoprotein levels in patients with psoriasis. Br J Dermatol. 1994; 130:738-737.

30. Azizzadeh M, Ghorbani R, Sharafi M. Serum lipids profiles in psoriatic patients. Koomesh. 2010;11(4(36)):307-312.

31. Mallbris L, Ritchlin C, Stahle M. Metabolic disorders in patients with psoriasis and psoriatic arthritis. Curr Rheumatol Rep. 2006;8:355-363.

32. Shahidi M, Javanbakht A, Darvish A. Lipid profile in psoriasis. Pajoohandeh. 2000;28:111-114.

33. Mebazaa A, El Asmi M, Zidi W, et al. Metabolic syndrome in Tunisian psoriatic patients: prevalence and determinants. J Eur Acad Dermatol Venereol. 2011;25(6):705-709. 
34. Naldi L, Chatenoud L, Linder D, et al. Cigarette smoking, body mass index, and stressful life events as risk factors for psoriasis: results from an Italian case-control study. J Invest Dermatol. 2005; 125(1):61-67.

35. Ashkevari S, Ehsani AH, Ghanbari A, Molaii H, Noormohammadpour P. The frequency of cigarette smoking in patients with psoriasis vulgaris: a comparative study. Tehran Univ Med J. 2011;64(4):260-266.

36. Gelfand JM, Neimann AL, Shin DB, Wang X, Margolis DJ, Troxel AB. Risk of myocardial infarction in patients with psoriasis. JAMA. 2006;296(14):1735-1741.

37. Jensen P1, Thyssen JP, Zachariae C, Hansen PR, Linneberg A, Skov L. Cardiovascular risk factors in subjects with psoriasis: a cross-sectional general population study. Int J Dermatol. 2013;52(6):681-683.

38. Zhu TY, Li EK, Tam LS. Cardiovascular risk in patients with psoriatic arthritis. Int J Rheumatol. 2012;2012:714321.
39. Armstrong AW, Harskamp CT, Armstrong EJ. The association between psoriasis and obesity: a systematic review and meta-analysis of observational studies. Nutr Diabetes. 2012;2:e54.

40. Cohen A, Weitzman D, Dreiher J. Psoriasis and hypertension: a casecontrol study. Acta Derm Venerol. 2010;90:23-26.

41. Ahlehoff O, Gislason G, Lindhardsen J, Skov L, Hansen PR. Patients with psoriasis have an increased risk of cardiovascular diseases. $U g e s k r$ Laeger. 2012;174(21):1450-1452.

42. Pereira RR, Amladi ST, Varthakavi PK. A study of the prevalence of diabetes, insulin resistance, lipid abnormalities, and cardiovascular risk factors in patients with chronic plaque psoriasis. Indian J Dermatol. 2011;56(5):520-526.

43. Koch M, Baurecht H, Ried J, et al. Psoriasis and cardiometabolic traits: modest association but distinct genetic architectures. J Investig Dermatol. 2015;135:1283-1293.

\section{Publish your work in this journal}

Clinical, Cosmetic and Investigational Dermatology is an international, peer-reviewed, open access, online journal that focuses on the latest clinical and experimental research in all aspects of skin disease and cosmetic interventions. All areas of dermatology will be covered; contributions will be welcomed from all clinicians and basic science researchers globally. This journal is indexed on CAS. The manuscript management system is completely online and includes a very quick and fair peer-review system, which is all easy to use. Visit http://www.dovepress.com/testimonials.php to read real quotes from published authors. 